\title{
Implications of anesthetic approach, spinal versus general, for the treatment of spinal disc herniation
}

\author{
Presented at the 2018 AANS/CNS Joint Section on Disorders of the Spine and Peripheral Nerves
}

\author{
Nikhil Sharma, BS, ${ }^{1}$ Matthew Piazza, MD, ${ }^{1}$ Paul J. Marcotte, MD, ${ }^{1,2}$ William Welch, MD,,2 \\ Ali K. Ozturk, MD, ${ }^{1,2}$ H. Isaac Chen, MD, ${ }^{1}$ Zarina S. Ali, MD, MS, ${ }^{1}$ James Schuster, MD, PhD, ${ }^{1,2}$ and \\ Neil R. Malhotra, MD ${ }^{1,2}$
}

\begin{abstract}
'Department of Neurosurgery, Hospital of the University of Pennsylvania, Philadelphia, Pennsylvania; and 2The Penn Spinal Research Group, Philadelphia, Pennsylvania
\end{abstract}

\begin{abstract}
OBJECTIVE Healthcare costs continue to escalate. Approaches to care that have comparable outcomes and complications are increasingly assessed for quality improvement and, when possible, cost containment. Efforts to identify components of care to reduce length of stay (LOS) have been ongoing. Spinal anesthesia (SA), for select lumbar spine procedures, has garnered interest as an alternative to general anesthesia (GA) that might reduce cost and in-hospital LOS and accelerate recovery. While clinical outcomes with SA or GA have been studied extensively, few authors have looked at the cost-analysis in relation to clinical outcomes. The authors' objectives were to compare the clinical perioperative outcomes of patients who received SA and GA, as well as the direct costs associated with each modality of care, and to determine which, in a retrospective analysis, can serve as a dominant procedural approach.
\end{abstract}

METHODS The authors retrospectively analyzed a homogeneous surgical population of 550 patients who underwent hemilaminotomy for disc herniation and who received either $S A(n=91)$ or $G A(n=459)$. All clinical and billing data were obtained via each patient's chart and the hospital's billing database, respectively. Additionally, the authors prospectively assessed patient-reported outcome measures for a subgroup of consecutively treated patients $(n=75)$ and compared quality-adjusted life year (QALY) gains between the two cohorts. Furthermore, the authors performed a propensity score-matching analysis to compare the two cohorts $(n=180)$.

RESULTS Direct hospital costs for patients receiving SA were $40 \%$ higher, in the hundreds of dollars, than for patients who received GA ( $p<0.0001)$. Furthermore, there was a significant difference with regard to LOS ( $p<0.0001)$, where patients receiving SA had a considerably longer hospital LOS (27.6\% increase in hours). Patients undergoing SA had more comorbidities $(p=0.0053)$, specifically diabetes and hypertension. However, metrics of complications, including readmission ( $p=0.3038$ ) and emergency department (ED) visits at 30 days $(p=1.0)$, were no different. Furthermore, in a small pilot group, QALY gains were statistically no different $(n=75, p=0.6708)$. Propensity score-matching analysis demonstrated similar results as the univariate analysis: there was no difference between the cohorts regarding 30-day readmission ( $p=1.0000$ ); ED within 30 days could not be analyzed as there were no patients in the SA group; and total direct costs and LOS were significantly different between the two cohorts $(p<0.0001$ and $p=0.0126$, respectively).

CONCLUSIONS Both SA and GA exhibit the qualities of a good anesthetic, and the utilization of these modalities for lumbar spine surgery is safe and effective. However, this work suggests that SA is associated with increased LOS and higher direct costs, although these differences may not be clinically or fiscally meaningful.

https://thejns.org/doi/abs/10.3171/2018.7.SPINE18460

KEYWORDS spinal anesthesia; general anesthesia; lumbar surgery; hemilaminotomy

$\mathrm{H}$ EALTHCARE costs have risen tremendously in the United States over the past decade, specifically with regard to care of degenerative spine conditions. ${ }^{9,11}$ A major contributor to increased cost of care is hospital length of stay (LOS). ${ }^{11,15}$ Efforts to identify modi- fiable components of perioperative and intraoperative care to reduce patient LOS and cost of care have been ongoing. ${ }^{10}$ In particular, spinal anesthesia (SA) for select lumbar spine procedures has garnered interest as a potential alternative anesthetic modality to streamline perioperative care.

ABBREVIATIONS ED = emergency department; GA = general anesthesia; $L O S=$ length of stay; $O R=$ operating room; $S A=$ spinal anesthesia SUBMITTED April 16, 2018. ACCEPTED July 19, 2018. 
Both SA and general anesthesia (GA) have been utilized successfully and safely in lumbar spine surgery.,2, 5,7 Despite the lack of evidence supporting one modality's clear superiority over the other, there is evidence to support advantages for each. SA provides the advantage of reduced postoperative pain, nausea, and vomiting, ,,4-7,12-14 while GA allows for prolonged procedures in the prone position, without compromising the airway, in addition to increased acceptance by patients. ${ }^{1,2,5,7}$ While clinical outcomes with SA or GA have been studied extensively using metrics involving the EQ-5D questionnaire (mobility, selfcare, pain, and the ability to carry out normal activities), few authors have performed a cost-analysis in relation to clinical outcomes. ${ }^{1,15}$

In the present study, we retrospectively analyzed data from a homogeneous cohort of patients who underwent lumbar spine surgery and who received either SA or GA. Our objectives were to compare the clinical perioperative outcomes related to patients who received SA and GA and to examine direct costs associated with care.

\section{Methods}

In this retrospective study, approved by the Institutional Review Board at the University of Pennsylvania, 550 patients were identified, using Current Procedural Terminology codes. Selected patients had undergone mostly singlelevel unilateral hemilaminotomy procedures (codes 63030 and 63035) for disc herniation between September 2013 and February 2017. Revision surgeries were excluded. Interventions that included more extensive surgery, beyond 1- to 2-level hemilaminotomy, were excluded. Patients were divided into two cohorts for comparative analysis: those receiving SA $(n=91)$ and those receiving GA $(n$ $=459$ ). All analyzed clinical data were obtained via the patient's electronic medical records or the hospital database, while all cost data were obtained from the hospital's billing database. Metrics obtained included age, race, sex, American Society of Anesthesiologists physical status grade, body mass index, medical comorbidities, smoking habits, hospital LOS, total direct costs, readmission within 30 days, and admission to the emergency department (ED) within 30 days. Total direct costs included all direct hospital costs (i.e., supplies) related directly to each patient, not charges. Costs accumulated for an individual patient from the time of entry to time of discharge and did not include historically highly variable estimates of indirect costs such as staffing or space. In a subsequent extension of the work, a prospective subgroup of consecutively treated patients $(n=75)$ completed outcome surveys, where quality gains were analyzed. Quality gains were determined utilizing the EQ-5D pre- and postoperatively for self-assessment. In addition to univariate regression, we performed a propensity score-matching analysis $(\mathrm{n}=180)$. Propensity scores were based on Charlson Comorbidity score, body mass index, and diabetes status and the caliper width calculated. Caliper width was set to 0.2 and multiplied by the standard deviation of the propensity score logits (0.071133). All patients undergoing surgery with SA were matched on a 1-to-1 basis with those undergoing surgery with GA on the propensity scores using the caliper width.
Chi-square test, t-test, Wilcoxon test, and KruskalWallis test were applied where appropriate to compare the two cohorts of patients for any significant differences. Statistical significance was considered if the calculated $\mathrm{p}$ value was $<0.05$. Hospital LOS and the direct cost of care during the index encounter associated with SA versus GA were the primary outcome measures. Secondary outcomes included 30-day readmission and admission to ED within 30 days.

\section{Results}

In the present study, 550 patients were enrolled who underwent lumbar spine surgery (459 patients received GA and 91 patients received SA). The study group included 244 females (44.4\%); the mean age for GA patients was 46.8 years (range 17-81 years) and that for those receiving SA was 45.9 years (range 17-79 years). Baseline characteristics were statistically comparable (Table 1). Patients undergoing surgery with SA had more medical comorbidities $(p=0.0053)$ than those undergoing surgery with GA; specifically, diabetes and hypertension were more common. Patients undergoing surgery with GA were more likely to undergo more complex procedures (i.e., multilevel discectomy) than those undergoing surgery with SA $(p=0.0249)$. There was a significant difference when considering hospital LOS $(\mathrm{p}<0.0001)$, where SA patients had a considerably longer hospital LOS (336.1 minutes) than GA patients (263.5 minutes), a $27.6 \%$ increase. The total direct cost was observed to be significantly different between the two cohorts $(p<0.0001)$. Patients undergoing lumbar spine surgery with SA had direct hospital costs

TABLE 1. Study population demographics

\begin{tabular}{|c|c|c|c|}
\hline \multirow[b]{2}{*}{ Variable } & \multicolumn{2}{|c|}{ Surgical Group } & \multirow[b]{2}{*}{ Total/p Value } \\
\hline & GA & SA & \\
\hline \multicolumn{4}{|l|}{ Sex, no. } \\
\hline Male & $225(49.02 \%)$ & $51(56.04 \%)$ & $306(55.6 \%)$ \\
\hline Female & $204(44.44 \%)$ & $40(43.96 \%)$ & $244(44.36 \%)$ \\
\hline Total & $459(83.45 \%)$ & $91(16.55 \%)$ & $550(100 \%)$ \\
\hline \multicolumn{4}{|l|}{ Race, no. } \\
\hline Black & $27(5.88 \%)$ & $3(3.29 \%)$ & $30(5.45 \%)$ \\
\hline White & $385(83.88 \%)$ & $78(85.71 \%)$ & $463(84.18 \%)$ \\
\hline Unknown & $14(3.05 \%)$ & $5(5.49 \%)$ & $19(3.45 \%)$ \\
\hline Other & $33(7.19 \%)$ & $5(5.51 \%)$ & $38(6.92 \%)$ \\
\hline Total & $459(83.45 \%)$ & $91(16.55 \%)$ & $550(100 \%)$ \\
\hline \multicolumn{4}{|l|}{ Age, yrs } \\
\hline Mean & 46.8 & 45.9 & \\
\hline SD & 13.8 & 14.0 & \\
\hline SE & 0.64 & 1.47 & \\
\hline Maximum & 81 & 79 & \\
\hline Minimum & 17 & 17 & \\
\hline \multicolumn{4}{|l|}{ Comorbidity } \\
\hline Diabetes, no. & $5(1.09 \%)$ & $4(4.40 \%)$ & $p=0.0455$ \\
\hline Hypertension, no. & $34(7.41 \%)$ & $16(17.58 \%)$ & $p=0.0044$ \\
\hline Overall (mean score) & 270.54 & 300.51 & $p=0.0053$ \\
\hline
\end{tabular}


TABLE 2. Overall differences between patients who received SA versus GA

\begin{tabular}{lccc}
\hline \multirow{2}{*}{ Variable Tested } & \multicolumn{2}{c}{ Surgical Group } & \\
\cline { 2 - 3 } & GA & SA & p Value \\
\hline Overall comorbidities, mean score & 270.54 & 300.51 & 0.0164 \\
\hline QALY gain, pilot cohort mean score & 37.32 & 39.74 & 0.6708 \\
\hline LOS, mins & 263.5 & 336.1 & 0.0001 \\
\hline Total direct costs, relative cost & $\mathrm{X}$ & $1.42 X$ & 0.0001 \\
\hline 30-day readmission, no. & 1 & 1 & 0.3017 \\
\hline ED visits w/in 30 days & 5 & 0 & 1.0 \\
\hline No. of levels intervened, mean score & 343 & 63.3 & 0.0249 \\
\hline
\end{tabular}

$Q A L Y=$ quality-adjusted life year.

$\mathrm{X}$ signifies a dollar amount; internal finance wanted the authors to share a ratio rather than true dollar amounts, which they believe are proprietary.

that were $42 \%$ higher, in hundreds of dollars, than those of their GA counterparts. Metrics of complications, including 30-day readmission $(\mathrm{p}=0.3038)$ and $\mathrm{ED}$ visits at 30 days $(\mathrm{p}=1.0)$, were no different. All statistical findings are summarized in Table 2.

Furthermore, in a small pilot group, quality-adjusted life year analysis demonstrated statistically similar global healthcare-related quality-of-life outcomes $(n=75, p=$ $0.6708)$. In this cohort, 54 patients received GA while 21 patients received SA (Table 2). Finally, in order to assess the differences in the two populations, propensity scorematching analysis $(\mathrm{n}=180)$ was performed, which demonstrated results consistent with the previous regression analysis. In this cohort, 90 patients were analyzed for each group. The 30-day readmission was no different between the two groups $(\mathrm{p}=1.000)$; it was not possible to determine ED admission within 30 days as no SA patients went to the ED. Direct costs were significantly different between the two cohorts $(\mathrm{p}<0.0001)$; SA patients had direct hospital costs that were $40 \%$ higher, in hundreds of dollars, than those of their GA counterparts. LOS, in hours and days, was also significantly different between the two groups $(p=0.0126)$, with SA patients staying longer than GA patients. All statistical findings relating to the propensity score-matching analysis are summarized in Table 3 (demographic data) and Table 4 (overall differences).

\section{Discussion}

Both SA and GA exhibit the qualities of a good anesthetic, and the utilization of these modalities for lumbar spine surgery is safe and effective. The data here suggest that while GA is favored, in terms of retrospective cost and LOS factors, the differences are likely not meaningful, and modality selection should be made based on hospital and physician skill set and preferences. The factors indicating a good anesthetic involve having a rapid onset and a rapid reversal of influence. Both SA and GA exhibit these qualities. ${ }^{1,2,4,13}$ However, there is a lack of data regarding the implications of the cost benefit of either modality.

To our knowledge, only two other studies have compared total cost associated with care directly between patients who received SA and GA..$^{1,15}$ In the present study,
TABLE 3. Propensity score-matched demographics

\begin{tabular}{|c|c|c|c|}
\hline \multirow[b]{2}{*}{ Variable } & \multicolumn{2}{|c|}{ Surgical Group } & \multirow[b]{2}{*}{ Total/p Value } \\
\hline & GA & SA & \\
\hline \multicolumn{4}{|l|}{ Sex } \\
\hline Male & $32(35.56)$ & $50(55.56)$ & $82(45.56)$ \\
\hline Female & $58(64.44)$ & $40(44.44)$ & $98(54.44)$ \\
\hline Total & $90(50)$ & $90(50)$ & 180 \\
\hline \multicolumn{4}{|l|}{ Race } \\
\hline Black & $6(6.67)$ & $3(3.33)$ & $9(5.0)$ \\
\hline White & 78 (86.67) & $77(85.56)$ & $155(86.11)$ \\
\hline Unknown & $4(4.44)$ & $5(5.56)$ & $9(5.0)$ \\
\hline Other & $2(2.22)$ & $5(5.56)$ & 7 (3.89) \\
\hline Total & 90 & 90 & 180 \\
\hline \multicolumn{4}{|l|}{ Age, yrs } \\
\hline Mean & 45.10 & 45.7 & \\
\hline SD & 13.93 & 13.94 & \\
\hline SE & 1.47 & 1.47 & \\
\hline Maximum & 80 & 79 & \\
\hline Minimum & 19 & 17 & \\
\hline \multicolumn{4}{|l|}{ Comorbidity } \\
\hline Diabetes & $4(4.44)$ & $4(4.44)$ & $p=1.0000$ \\
\hline Hypertension & $9(10.0)$ & $15(16.67)$ & $p=0.1883$ \\
\hline
\end{tabular}

Values are presented as the number (\%) of patients unless otherwise indicated.

GA for patients undergoing lumbar microdiscectomy predicted lower direct costs and shorter LOS as compared to similar patients undergoing surgery with SA. Furthermore, short-term clinical outcomes were no different based on anesthesia type. The current literature, however, shows conflicting results. Agarwal et al. retrospectively analyzed a population of 542 patients undergoing a heterogeneous lumbar surgical intervention (elective, noninstrumented lumbar spine surgery) and found that SA patients had lower total costs by $39.6 \%$ when compared to GA patients. ${ }^{1}$ However, the costs included estimated operating room (OR) utilization costs, which are theoretically less with SA because recovery occurs outside the OR. Walcott et al. retrospectively analyzed a 400-patient population undergoing a grouping of heterogeneous lumbar interven-

TABLE 4. Propensity score matched: overall differences between patients who received SA versus GA

\begin{tabular}{lccr}
\hline \multirow{2}{*}{ Variable } & \multicolumn{2}{c}{ Surgical Group } & \\
\cline { 2 - 3 } & $\mathrm{GA}$ & $\mathrm{SA}$ & $\mathrm{p}$ Value \\
\hline Overall comorbidities, mean score & 10.14 & 15.54 & 0.4079 \\
\hline LOS, hrs & 20.44 & 30.84 & 0.0126 \\
\hline Total direct costs, relative cost & $\mathrm{X}$ & $1.40 \mathrm{X}$ & $<0.0001$ \\
\hline 30-day readmission, no. & 1 & 1 & 1.0000 \\
\hline ED visits w/in 30 days, no. & 0 & 1 & $\mathrm{NA}$ \\
\hline
\end{tabular}

$\mathrm{NA}=$ not applicable.

$X$ signifies a dollar amount; internal finance wanted the authors to share a ratio rather than true dollar amounts, which they believe are proprietary. 
tions similar to the population described by Agarwal et al. (elective, noninstrumented lumbar spine surgery) and studied direct OR costs rather than including indirect estimates. They found that direct operating room costs were $10.33 \%$ lower for SA patients than for GA patients..$^{15}$ They concluded that although SA and GA had comparable complication rates, SA had a financial benefit associated with its use. ${ }^{15}$ Agarwal et al. and Walcott et al., however, studied heterogeneous populations in terms of the procedure that was performed (discectomy, laminectomy, etc.), and more complex surgical approaches appear to be weighted toward the GA group. While both author groups provided extremely valuable conclusions about a subset of surgical patients, our goal was to focus on a very homogeneous surgical population and total direct costs for the hospital visit, rather than one component of the hospitalization. It is our hope that our results might provide surgeons with a clear, albeit retrospective, guide for a very specific patient population undergoing a common procedure. In an effort to limit the impact of non-anesthesia-related variables, our study focused exclusively on a homogeneous population from the perspective of the procedure performed (lumbar discectomy) and solely on direct costs, and our conclusions reflect as such.

LOS differed between times reported in the current literature and the time observed in the present study. Chen et al. observed no significant differences regarding patient demographics or operative times; however, they did observe shorter hospital LOS (2.96 days vs 3.83 days, respectively) and a higher surgical success rate for SA patients than for GA patients (97\% for SA vs $94 \%$ for GA). ${ }^{3}$ Similarly, McLain et al. concluded that SA reduced overall hospital LOS, increased patient satisfaction, and required less pain medication. ${ }^{8}$ A shorter hospital LOS for SA patients is in contrast to our observation in the present study, as our analysis indicated a shorter LOS for GA patients than for SA patients (263.5 minutes vs 336.1 minutes); however, the overall LOSs in our cohorts were dramatically shorter than those in previously published work. The paradigm shift toward outpatient care of these patients and procedures makes the comparison LOS to older published cohorts challenging.

Regarding the superiority of one modality over the other, there seems to be a polarized divide in the current literature. There are many studies documenting advantages of SA over GA in terms of postoperative clinical metrics such as decreased postoperative nausea, shorter hospital LOS, shorter duration of anesthesia and surgery, and lower estimated blood loss. ${ }^{1-4}$ However, in a prospective randomized trial of 100 patients undergoing laminectomy for lumbar disc herniation, Sadrolsadat et al. demonstrated that there were in fact no significant advantages to SA over GA and, furthermore, that GA may reduce complications,${ }^{13}$ including fewer instances of intraoperative hypotension and postoperative nausea and vomiting. Attari et al. concluded that because of its lower intraoperative blood loss and increased surgeon satisfaction, SA should be considered the superior modality over GA. ${ }^{2}$ De Rojas et al. concluded that SA should be recommended for older patients, as there are more short-term benefits such as decreased analgesic requirements, decreased postoperative nausea, and a more favorable hemodynamic outcome. ${ }^{4}$ Similarly, Demirel et al. demonstrated that SA showed reduced operative times and blood loss while having favorable hemodynamic outcomes and improved short-term postoperative outcomes. ${ }^{5}$ In the present study, we saw that differences between the two cohorts were significant, although perhaps not clinically meaningful. Patients receiving SA had statistically significantly higher direct costs and longer LOS, but the differences were in the hundreds-of-dollars range and time changes measured in minutes, not days.

This study had certain limitations. Since it was retrospective in nature, there is potential for selection bias and incomplete medical records. However, we performed a propensity score-matching analysis to ensure that the results were unchanged. Additionally, the current study only looked at direct costs, while indirect and variable costs could alter the findings. Furthermore, the present study only examined perioperative variables, not long-term cost. However, we do not expect differing long-term cost implications related to anesthetic type. Despite statistically significant findings, additional prospective randomized studies will be needed to further validate these conclusions given the retrospective nature of this work. Despite similar clinical outcomes between the two cohorts, GA was associated with lower direct cost of care than SA. This conclusion can be utilized in a clinical setting to assist in decreasing the cost of care to patients undergoing lumbar surgery while providing similar quality of care.

\section{Conclusions}

In the present study, higher total direct costs and longer hospital LOS were associated with patients receiving SA in both matched and unmatched analysis. In a small prospective pilot, healthcare-related quality-of-life outcomes were not impacted by anesthesia type, despite overall outcomes being similar. Additionally, propensity scorematching analysis demonstrated similar results to those of the univariate regression analysis. Based on the analysis in this study, we conclude that GA has statistically significant advantages over SA for patients undergoing lumbar discectomy; however, these advantages may not be clinically meaningful given the low dollar amounts and LOS differences. Thus, we conclude that either technique may be considered to be reasonable, given the low overall direct costs and LOS.

\section{Acknowledgments}

We thank the Bernadette and Kevin Mckenna Family Fund for providing financial support and the Neurosurgery Quality Improvement Initiative (NQII) EpiLog project for providing data.

\section{References}

1. Agarwal P, Pierce J, Welch WC: Cost analysis of spinal versus general anesthesia for lumbar diskectomy and laminectomy spine surgery. World Neurosurg 89:266-271, 2016

2. Attari MA, Mirhosseini SA, Honarmand A, Safavi MR: Spinal anesthesia versus general anesthesia for elective lumbar spine surgery: a randomized clinical trial. J Res Med Sci 16:524-529, 2011

3. Chen HT, Tsai CH, Chao SC, Kao TH, Chen YJ, Hsu HC, et 
al: Endoscopic discectomy of L5-S1 disc herniation via an interlaminar approach: prospective controlled study under local and general anesthesia. Surg Neurol Int 2:93, 2011

4. De Rojas JO, Syre P, Welch WC: Regional anesthesia versus general anesthesia for surgery on the lumbar spine: a review of the modern literature. Clin Neurol Neurosurg 119:3943, 2014

5. Demirel CB, Kalayci M, Ozkocak I, Altunkaya H, Ozer Y, Acikgoz B: A prospective randomized study comparing perioperative outcome variables after epidural or general anesthesia for lumbar disc surgery. J Neurosurg Anesthesiol 15:185-192, 2003

6. Jellish WS, Thalji Z, Stevenson K, Shea J: A prospective randomized study comparing short- and intermediate-term perioperative outcome variables after spinal or general anesthesia for lumbar disk and laminectomy surgery. Anesth Analg 83:559-564, 1996

7. Kahveci K, Doger C, Ornek D, Gokcinar D, Aydemir S, Ozay $\mathrm{R}$ : Perioperative outcome and cost-effectiveness of spinal versus general anesthesia for lumbar spine surgery. Neurol Neurochir Pol 48:167-173, 2014

8. McLain RF, Bell GR, Kalfas I, Tetzlaff JE, Yoon HJ: Complications associated with lumbar laminectomy: a comparison of spinal versus general anesthesia. Spine (Phila Pa 1976) 29:2542-2547, 2004

9. Mok JM, Martinez M, Smith HE, Sciubba DM, Passias PG, Schoenfeld A, et al: Impact of a bundled payment system on resource utilization during spine surgery. Int J Spine Surg 10:19, 2016

10. Puffer RC, Planchard R, Mallory GW, Clarke MJ: Patientspecific factors affecting hospital costs in lumbar spine surgery. J Neurosurg Spine 24:1-6, 2016

11. Rossi VJ, Ahn J, Bohl DD, Tabaraee E, Singh K: Economic factors in the future delivery of spinal healthcare. World $\mathbf{J}$ Orthop 6:409-412, 2015

12. Rung GW, Williams D, Gelb DE, Grubb M: Isobaric spinal anesthesia for lumbar disk surgery. Anesth Analg 84:11651166, 1997
13. Sadrolsadat SH, Mahdavi AR, Moharari RS, Khajavi MR, Khashayar P, Najafi A, et al: A prospective randomized trial comparing the technique of spinal and general anesthesia for lumbar disk surgery: a study of 100 cases. Surg Neurol 71:60-65, 2009

14. Tetzlaff JE, Dilger JA, Kodsy M, al-Bataineh J, Yoon HJ, Bell GR: Spinal anesthesia for elective lumbar spine surgery. J Clin Anesth 10:666-669, 1998

15. Walcott BP, Khanna A, Yanamadala V, Coumans JV, Peterfreund RA: Cost analysis of spinal and general anesthesia for the surgical treatment of lumbar spondylosis. J Clin Neurosci 22:539-543, 2015

\section{Disclosures}

Dr. Welch reports owning stock in Transcendental Spine.

\section{Author Contributions}

Conception and design: Malhotra, Sharma. Acquisition of data: Malhotra, Sharma, Piazza. Analysis and interpretation of data: Malhotra, Sharma, Piazza. Drafting the article: Malhotra, Sharma, Piazza. Critically revising the article: all authors. Reviewed submitted version of manuscript: all authors. Approved the final version of the manuscript on behalf of all authors: Malhotra. Study supervision: Malhotra.

\section{Supplemental Information Previous Presentations}

Portions of this work were presented at the 34th Annual Meeting of the AANS/CNS Joint Section on Disorders of the Spine and Peripheral Nerves, March 14-17, 2018, Orlando, FL.

\section{Correspondence}

Neil R. Malhotra: University of Pennsylvania, Philadelphia, PA. nrm@uphs.upenn.edu. 